\title{
Jamming in Hierarchical Networks
}

\author{
Xiang Cheng and Stefan Boettcher* \\ Department of Physics, Emory University, Atlanta, GA 30322, USA
}

\begin{abstract}
We study the Biroli-Mezard model for lattice glasses on a number of hierarchical networks. These networks combine certain lattice-like features with a recursive structure that makes them suitable for exact renormalization group studies and provide an alternative to the mean-field approach. In our numerical simulations here, we first explore their equilibrium properties with the WangLandau algorithm. Then, we investigate their dynamical behavior using a grand-canonical annealing algorithm. We find that the dynamics readily falls out of equilibrium and jams in many of our networks with certain constraints on the neighborhood occupation imposed by the Biroli-Mezard model, even in cases where exact results indicate that no ideal glass transition exists. But while we find that time-scales for the jams diverge, our simulations can not ascertain such a divergence for a packing fraction distinctly above random close packing. In cases where we allow hopping in our dynamical simulations, the jams on these networks generally disappear.
\end{abstract}

Keywords. Jamming transition, Wang-Landau sampling, Monte Carlo simulation, hierarchical network

\section{INTRODUCTION}

The jamming transition, as discussed by Liu and Nagel in 1998 [1], for example, has been the focus of both theoretical and experimental studies [2-4]. A granular disordered system for increasing density can reach a jammed state at which a finite yield stress develops [1, 5], or at least extremely long relaxation times ensue [6, 7], similar to the emerging sluggish behavior observed when the viscosity of a cooled glassy liquid seemingly diverges. Thus, a jamming transition may be induced in various ways, such as by increasing density, decreasing temperature, or/and reducing shear stress [3]. Below the jamming transition, the system stays in long-lived meta-stable states, and its progression to its corresponding equilibrium state entails an extremely slow, non-Debye relaxation [8-11]. Jamming transitions have been observed in various types of systems, such as granular media [12], molecular glasses [13, 14], colloids [15], emulsions [16], foams [17, 18], and some theoretical systems [4, 11, 19]. These systems can behave like stiff solids at a high density with low temperature and small perturbations. In these transitional processes, the systems can self-organize their own structure to avoid large fluctuations [17] and to reach a quasi-stable jammed state, characterized by an extremely slow evolution to the unjammed equilibrium state. Some studies show that the sluggishness may indicate the existence of a Gardner transition in which the energy landscape contains numerous local minima separated by high barriers [20, 21]. The properties of those quasi-stable non-equilibrium states as well as their corresponding equilibrium state is the main focus of this paper.

The properties of the jamming transition have been studied extensively $[2-4,12]$, but we still lack an essen-

* Corresponding authors email addresses: xcheng7@emory.edu (Xiang Cheng), sboettc@emory.edu (Stefan Boettcher) tial understanding of the physics underlying the jammed state. In particular, it is still an open question whether the onset of jamming itself is associated with any (or several [19]) phase transitions, and whether any such transition in the dynamics would be associated with emerging features of the energy landscape in configuration space $[20,22]$. The later would indicate the possibility of linking jamming with an underlying equilibrium transition. Despite of much progress in simulating computational models of jamming, theoretical progress has been much slower than the accumulation of experimental discoveries. One of the reasons is the scarcity of theoretical models with a microscopically tractable thermodynamics that capture the complex jamming process [23, 24]. In recent years, a lattice glass model proposed by Biroli and Mezard (BM) [25] has been shown as a simple but adequate means to study the jamming process in such detail. It is simple because the model follows specific dynamical rules which are elementary to implement in both simulations and analytical work. In distinction to kinetically constrained models such as that due to Kob and Andersen [26], in which particles are blocked from leaving a position unless certain neighborhood conditions are satisfied, BM embeds geometric frustration merely by preventing the neighborhood of any particles to consist of more than $l$ other particles. Beyond that, it proceeds purely thermodynamically. The phase diagram can be reduced to just one (or both) of two control parameters, chemical potential and temperature. Either is sufficient to reproduce a jamming transition which is similar to that observed in off-lattice systems [25].

Using the BM-model in a mean-field network (i.e., a regular random graph), Krzakala et al. find jammed states in Monte Carlo simulations and a genuine thermodynamical phase transition (ideal glass transition) in its mean-field analytical solutions [23]. In other words, the jammed state coincides with an underlying equilibrium state that possesses a phase transition to a glassy state. Also, other recent studies in different glassy systems indicate a close relationship between the jamming

(C) 2015. This manuscript version is made available under the Elsevier user license

http://www.elsevier.com/open-access/userlicense/1.0/ 
transition and other phase transitions, such as random first-order transition [27], glass transition [19, 28], and Gardner transition [20]. These investigations strongly indicate that a true phase transitions might be the reason for the onset of jamming. The theoretical evidence for such a connection thus far is based largely on meanfield models [20, 22, 23, 25], as such a transition is hard to ascertain for finite-dimensional lattice glasses. Yet, it remains unclear whether mean-field solutions in disordered systems can provide an adequate conception for real-world behavior.

In this paper, we propose to use the lattice glass model BM on hierarchical networks $[29,30]$, which are small-world networks with a fixed, lattice-like geometry. They combine a finite-dimensional lattice backbone with a hierarchy of small-world links that in themselves impose a high degree of geometric frustration despite of their regular pattern. These networks can be considered as an instructive intermediary between mean-field and finite-dimensional lattice system, which may reveal more insights about real systems. Their recursive patterns and fixed structures make them computationally approachable and analytically solvable using renormalization group (RG). Moreover, these networks have been shown to possess interesting phase transitions in the Ising model [31] or for percolation [32, 33], but no glassy systems have been studied on these networks. It would be interesting to see how these networks perform in the lattice glass model. More details of why using these networks are discussed in Sec.III.B.

Our goal in this work is to find (1) whether the lattice glass model leads to jamming states in hierarchical networks, (2) whether there is an ideal glass transition underlying the jamming transition, and (3) whether the local dynamics affects the jamming process. To our knowledge, these questions have not been studied in any small-world systems. Our results can contribute new insights to understand jamming.

We find that BM in these networks can jam, even when there is certifiably no equilibrium transition [30]; the geometric frustration that derives from the incommensurability among the small-world links is sufficient in many cases to affect jamming. In fact, jamming is most pronounced for fully exclusive neighborhoods $(l=0)$. It disappears for more disordered neighborhoods $(l=1)$, at least for our non-regular networks, where the allowance of $l=1$ neighbor to be occupied seems to provide the "lubrication" that averts jams. However, the packing fractions at which time-scales diverge is virtually indistinguishable from random close packing within the accuracy of our simulations.

Mean-field calculations of BM in Ref. [22] predict a kinetic transition for dynamic rules based on nearestneighbor hopping. In our simulations, we find that such hopping, in addition to the particle exchange with a bath, can affect a dramatic change in the dynamic behavior and eliminated jamming in all cases we consider.

This paper is organized as follows. In Sec. II, we de- scribe the model, the networks, and our numerical simulations. In Sec. III we discuss the results of our simulations for each network. In Sec. IV we conclude with a few summary remarks and an outlook for future work.

\section{MODEL \& METHODS}

In this section, we describe the model and the networks on which we will study its behavior. To benchmark the equilibrium properties of the model on those networks, we implement a multi-canonical algorithm due to Wang and Landau $[34,35]$. We further need a grand-canonical annealing algorithm to study the dynamics of the lattice glass model on those networks.

\section{A. Lattice glass model}

The lattice glass model as defined by Biroli and Mezard (BM) [25] considers a system of particles on a lattice of $N$ sites. Each site can carry either $x_{i}=0$ or $x_{i}=1$ particle, and the occupation is restricted by a hard, local "density constraint": any occupied site $\left(x_{i}=1\right)$ can have at most $l$ occupied neighbors, where $l$ could range locally from 0 to the total number of its neighbor-sites. In this model, the jamming is defined thermodynamically by rejecting the configurations violating the density constraint. Here, we focus on global density constraints of $l=0$ (completely excluded neighborhood occupation) and $l=1$ as the most generic cases. The system can be described by the grand canonical partition function

$$
Z(\mu)=\sum_{\text {allowed }\left\{x_{i}\right\}} \exp \left[\mu \sum_{i=1}^{N} x_{i}\right]
$$

where the sum is over all the allowed configurations $\left\{x_{i}\right\}$. Here, $\mu$ is the reduced chemical potential, where we have chosen units such that the temperature is $k_{B} T=1 / \beta=$ 1 , and $\sum_{i=1}^{N} x_{i}$ is the total number of particles in a specific configuration.

From the grand canonical partition function in Eq. (1), we can obtain the thermodynamic observables we intend to measure, such as the Landau free energy density $w(\mu)$, the packing fraction $\rho(\mu)$, and the entropy density $s[\rho(\mu)]$, as defined in the following equations:

$$
\begin{aligned}
& w(\mu)=-\frac{1}{N} \ln Z, \\
& \rho(\mu)=\frac{1}{N}\left\langle\sum_{i=1}^{N} x_{i}\right\rangle_{\mu}=\frac{1}{N} \frac{\partial \ln Z}{\partial \mu}, \\
& s(\mu)=\frac{1}{N}\left(1-\mu \frac{\partial}{\partial \mu}\right) \ln Z .
\end{aligned}
$$




\section{B. Hierarchical networks}

In our investigations, the 3 hierarchical networks are the Hanoi networks [29]. They are small-world networks with a hierarchical, recursive structure built on a 1D lattice. There are 4 main reasons of using these hierarchical networks:

1. The recursive nature of the patterns can ultimately provide analytical solution via the renormalization group (RG) [30], which is usually impossible for most fixed-structure networks;

2. Their lattice-like structures share more similarities with regular lattices than mean-field models, which could contribute more insights about realworld systems than mean-field models;

3. These three hierarchical networks have different degrees and geometries from which we may learn the relationship between geometry and jamming;

4. The fixed structures, unlike a random geometry in many mean-field models, can significantly reduce the computational cost by avoiding averages over many random ensembles.

We use three Hanoi networks: HN3 is a network with a regular degree of 3 , while HN5 is a similar network that possesses many extra links such that vertices have an exponential arrangement of degrees with an average degree of 5. HNNP is a similar Hanoi network of average degree of 4, but which is non-planar. Each of them can be built on a simple backbone of a 1D lattice. The 1D backbone has $N=2^{k}+1(k=1,2,3, \cdots)$ sites where each site is numbered from 0 to $N$. Any site $n, 0<n \leq N$, can be defined by two unique integers $i$ and $j$,

$$
n(i, j)=2^{i-1}(2 j+1),
$$

where $i, 1 \leq i \leq k$, denotes the level in the hierarchy and $j, 0 \leq \bar{j}<2^{\bar{k}-i}$, labels consecutive sites within each hierarchy $i$. Site $n=0$ is defined in the highest level $k$ or, equivalently, is identified with site $n=N$ for periodic boundary conditions. With this setup, we have a 1D backbone of degree 2 for each site and a well-defined hierarchy on which we can build long-range links recursively in three different ways: HN3 [29] is constructed by connecting the neighbor sites $n(i, 0) \longleftrightarrow n(i, 1)$, $n(i, 2) \longleftrightarrow n(i, 3), n(i, 4) \longleftrightarrow n(i, 5)$, and so on and so forth. For example, in level $i=1$, site $n(1,0)=1$ is connected to $n(1,1)=3$; site $n(1,2)=5$ is connected to $n(1,3)=7$; and so on. A initial section of a HN3 network is given in Fig. 1. As a result, HN3 is a planar network of regular degree 3 .

HN5 [32], as shown in Fig. 2, is an extension based on HN3, where each site in level $i$ ( $i \geq 2$, i.e., all even sites) is further connected to sites that are $2^{i-1}$ sites away in both directions. For example, for the level $i=2$ sites (sites $2,6,10, \cdots)$, site 2 is connected to both site 0 and site 4 ;

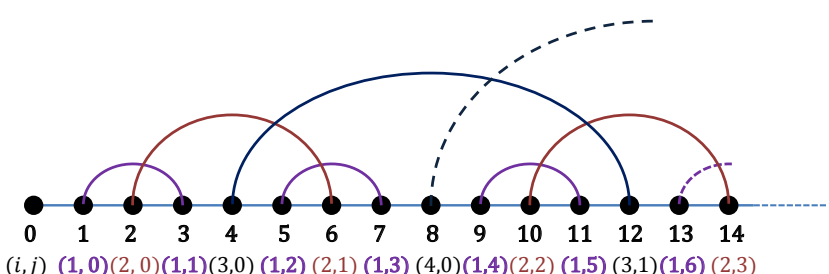

FIG. 1. An example of the first 14 sites of HN3 on a semiinfinite line.

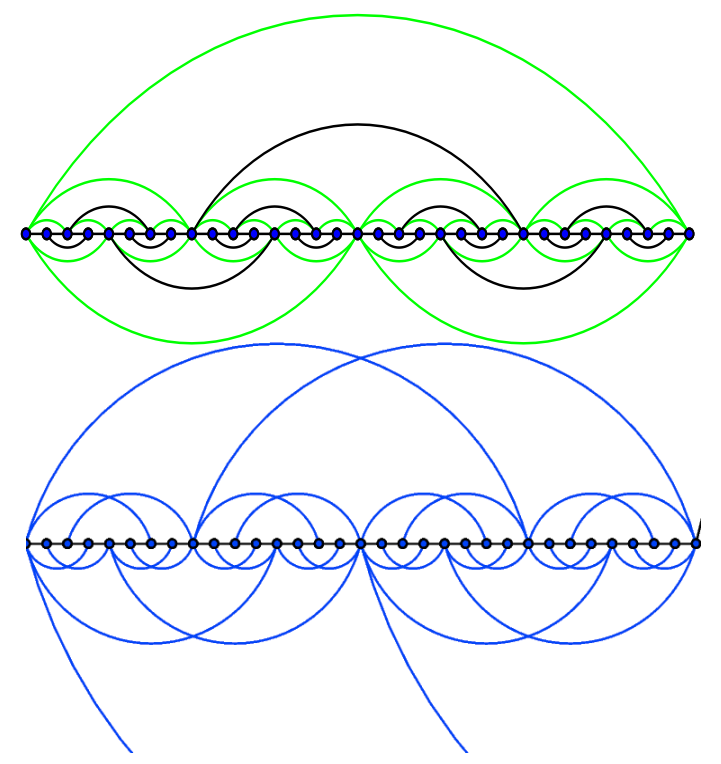

FIG. 2. Depiction of HN5 (top) and HNNP (bottom), first introduced in Ref. [32]. Green-shaded lines in HN5 represent its difference to HN3, which is at its core (dark lines). While HN3 and HN5 are planar, HNNP is non-planar.

site 6 is connected to sites 4 and 8; etc. The resulting network remains planar but has a hierarchy-dependent degree, i.e., $1 / 2$ sites have degree $3,1 / 4$ have degree 5 , $1 / 8$ have degree 7 , etc. In the limit of $N \rightarrow \infty$, this network has average degree 5 .

HNNP [32], also shown in Fig. 2, is constructed from the same 1D backbone as HN3 and HN5. However, for site $n$ in level $i$ with even $j$, it is connected forwards to site $\left(n+3 \times 2^{i-1}\right)$; while site $n$ in level $i$ with odd $j$ is connected backwards to site $\left(n-3 \times 2^{i-1}\right)$. Level 1 and level 2 sites have degree 3 , and level $3,4,5, \cdots$ sites have degree $5,7,9, \cdots$. The HNNP has an average degree of 4 and is non-planar.

\section{Wang-Landau Sampling}

Wang-Landau sampling [34] is a multi-canonical method to numerically determine the entire density of states $g_{n}$ within a single simulation. This method is based on the fact that a random walk in the configuration space with a probability proportional to the inverse 
of the density of states with occupation $n, 0 \leq n \leq N$, enforces a flat histogram in $g_{n}$ over all $n$. Based on this fact, Wang-Landau sampling keeps modifying the estimated density of states in the random walks over all possible configurations and can make the density of states converge to the true value. The update procedure is:

1. Initially, set all unknown density of states $\left\{g_{n}=\right.$ $1\}$ and the histogram $\left\{H_{n}=0\right\}$ for all occupations $n$, initiate the modification factor $f=e^{1} \approx$ $2.71828 \ldots$;

2. Randomly pick a site $i$; if it is empty (occupied), add (remove) a particle with a probability of $\min \left[1, \frac{g_{n}}{g_{n+1}}\right]\left(\min \left[1, \frac{g_{n}}{g_{n-1}}\right]\right)$ while obeying the rule of the hard local density constraint on having at most $l$ occupied nearest neighbors of site $i$;

3. Randomly pick one occupied site and one empty site; transfer a particle from the occupied site to the empty, if the density constraint is not violated;

4. Update the $H_{n}$ and $g_{n}$ of the current state, i.e., set $\left\{H_{n}=H_{n}+1\right\}$ and $\left\{g_{n}=g_{n} \times f\right\}$;

5. Repeat steps 2 to 4 until the sampling reaches a nearly flat histogram for the $H_{n}$, then update the modification factor $f=\sqrt{f}$ and reset $\left\{H_{n}=0\right\}$;

6. Stop if $f \leq 1+10^{-8}$.

Our procedure mostly follows the standard procedure of Wang-Landau sampling [34], except for step 3. Its purpose is to facilitate the random walk to explore phase space more broadly and to expedite convergence.

Wang-Landau sampling has been proved as an effective method to find the density of states [34, 36, 37]. In our study, it can find convergence for system size of up to $N \sim 10^{3}$ within a reasonably computational cost. From the density of states, we can calculate the equilibrium thermodynamical properties for the corresponding system sizes.

\section{Grand-Canonical Annealing}

In parallel to the equilibrium properties provided by Wang-Landau sampling, we also implement a form of simulated annealing [38] to explore the dynamics of the model and the possibility of jamming, in a process that is similar to an experiment. Simulated annealing used in this study follows the standard procedure [39], and similar setups are also used in Ref. [23] and [25]. The corresponding experiment is exchanging particles between the network and a reservoir of particles with (dimensionless) chemical potential $\mu$. In our study, the annealing speed is not controlled by decreasing temperature (which we set to $\beta=1$ ) but by increasing the chemical potential. The annealing algorithm is:

1. Initially, start with chemical potential $\mu_{0}=0$;
2. Randomly pick a site $n$; if it is empty (occupied), add (remove) a particle with a probability of $\min [1, \exp (\mu)](\min [1, \exp (-\mu)])$ while obeying the rule of the hard local density constraint on having at most $l$ occupied nearest neighbors of $n$;

3. If hopping is allowed, randomly pick one site; only if it is occupied, randomly pick one of its empty neighbor(s) and displace the particle if the density constraint remains satisfied;

4. Increase $\mu$ by $d \mu$ every 1 Monte Carlo sweep ( $N$ random updates), where $d \mu / d t$ (in time-units of $d t=1)$ is the annealing schedule and $d \mu \ll 1$;

5. Repeat steps 2 to 4 until $\mu$ reaches a certain (large) chemical potential.

Following the procedure above, the simulated annealing can reveal whether or not a jamming transition occurs in the process. Besides that, we can test the effect of local dynamics [23, 40] by adding a local hopping random walk (step 3), i.e., a particle can transfer any of its empty neighboring sites as long as the constraint remains satisfied. The results are shown and explained in the following section.

\section{RESULTS}

To assess the properties of jamming, we first have to benchmark our systems with the corresponding equilibrium behaviors. After that, we discuss the dynamic simulations with the annealing algorithm in reference to these equilibrium benchmarks.

\section{A. Equilibrium Properties}

Wang-Landau sampling, as described in Sec. II C, is ideally suited for our purpose, since it provides access directly to the density of states $g_{n}$ as a function of occupation number $n$, which yields the partition function as

$$
Z(\mu)=\sum_{n=0}^{n_{\max }} g_{n} e^{n \mu}
$$

All thermodynamic quantities in the equilibrium can be obtained numerically by summation of the formal derivates of $Z(\mu)$, such as those in Eqs. (2), over all permissible occupation numbers $0 \leq n \leq n_{\max }<N$. (For all $n_{\max }<n \leq N$ it is $g_{n}=0$.)

In Fig. 3, we plot the density of states as a function of the packing fraction, both obtained with Wang-Landau. It becomes apparent that each model has a simple rational value for its optimal $(\mu \rightarrow \infty)$ "random" close packing fraction $\rho_{C P}=n_{\max } / N$. This corresponds to a random packing in the sense that it has a nontrivial 
TABLE I. Closest packing fractions $\rho_{C P}$ found by WangLandau sampling. The values for $l=0$ have been previously obtained with exact RG, the one for HNNP being unique, with every second, odd site occupied. For $l=1$, we also predict exact fractions with nontrivial entropy densities, see Fig. 3.

\begin{tabular}{|c|c||c|}
\hline Network & $l=0$ & $l=1$ \\
\hline \hline HN3 & $3 / 8[30]$ & $9 / 16$ \\
\hline HN5 & $1 / 3[30]$ & $1 / 2$ \\
\hline HNNP & $1 / 2$ & $1 / 2$ \\
\hline
\end{tabular}

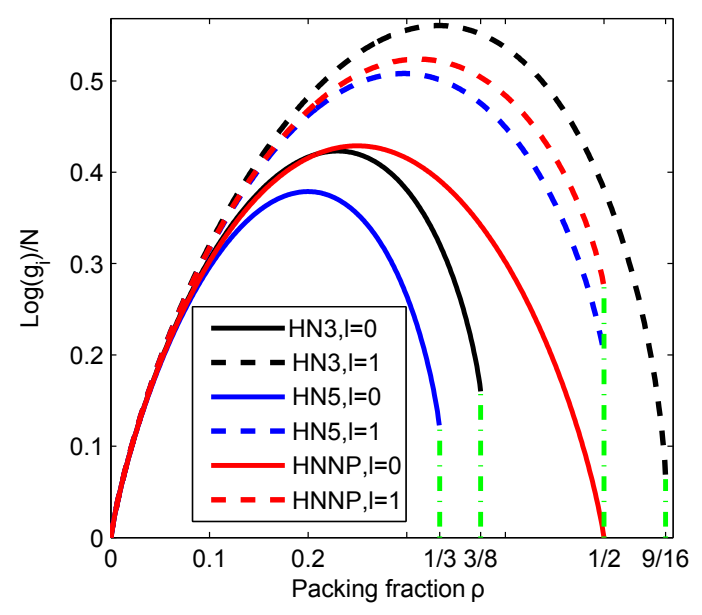

FIG. 3. Density of states from Wang-Landau sampling at $N=1024$. The green dash-dot vertical line are showing the closest packing fractions (as shown in Table I) for each system. Note that only for HNNP at $l=0$ there is a unique, crystalline ground state.

entropy density due to geometric disorder (imposed by the lack of translational invariance in the lattice), except for HNNP at $l=0$, which has a unique "crystalline" packing of every odd site being occupied. While these values for $\rho_{C P}$ have been previously obtained with $\mathrm{RG}$ for $l=0$ [30], the simulations predict also strikingly simple but nontrivial values for $l=1$, where exact $R G$ is likely not possible. These values are listed in Table I.

Wang-Landau sampling converges within a reasonable time for system sizes smaller than $N \approx 2000$ but fails to converge for larger system size. There may be two reasons for the lack of convergence: (1) the density of states is not symmetric as a function of packing fraction, and this asymmetry requires Wang-Landau to sample the whole configuration space, which increases the computational cost dramatically especially for large system sizes; (2) the lower the density of states of the closest packed state, the harder it is for Monte Carlo sampling to find its closest packing state because of the hard-density constraint. Although Wang-Landau sampling fails for large system sizes, the results of system size $N=1024$ can still offer an insight to the equilibrium state because the density of states and the packing fraction exhibit only
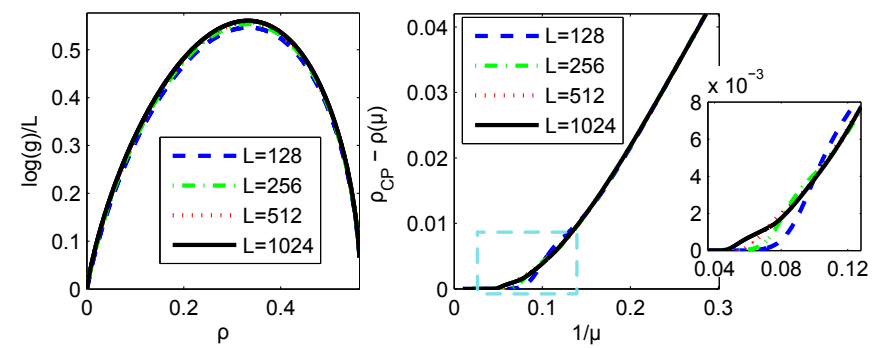

FIG. 4. Convergence to the thermodynamic limit for finite system sizes for the example of HN3 with $l=1$ using WangLandau sampling. The figures are for the density of states (left) and the packing fraction (right). The equilibrium packing fraction $\rho(\mu)$ as a function of chemical potential $\mu$ is calculated from the density of states according to Eq. (2); it approaches the closest packing fraction $\rho_{C P}$ for $1 / \mu \rightarrow 0$. The convergence for other systems is similar or better.

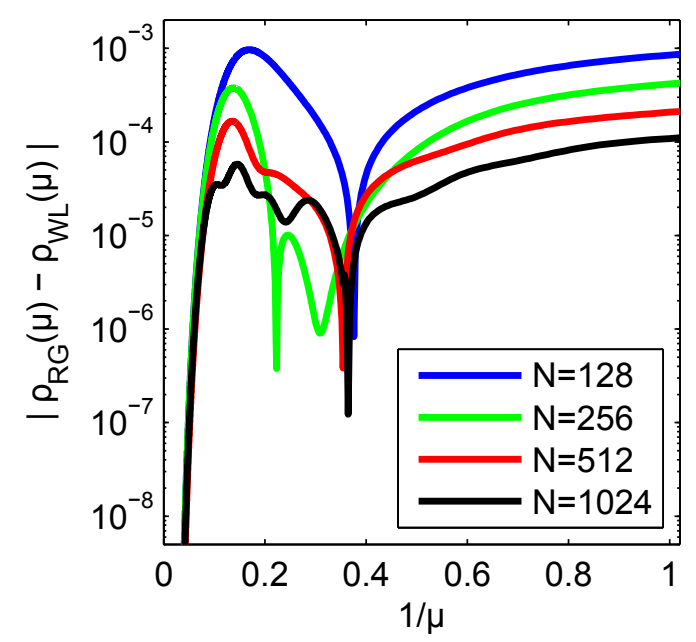

FIG. 5. Plot of the error in the finite-size packing fraction in Wang-Landau sampling, $\left|\rho_{W L}-\rho_{R G}\right|$, as a function of $1 / \mu$ near close packing $(\mu \rightarrow \infty)$ in HN3 at $l=0$. Here, RG result $\rho_{R G}(\mu)$ from Ref. [30] at system size $N=2^{500}$ is taken as the exact, thermodynamic packing fraction. Relative to $\rho_{R G}(\mu)$, the finite-size packing fraction, $\rho_{W L}(\mu)$, at $N=2^{k}$ with $k=$ $8,9,10$ already exhibit quite small and rapidly diminishing corrections.

small finite-size corrections for increasing $N$. For example, the convergence of HN3 with $l=0$ is shown in Fig.4. Other networks with $l=0,1$ have similar or even better convergence.

We can further demonstrate the quality of the WangLandau simulations, and appraise their residual finitesize effects, by comparison with exact results obtained with the renormalization group $(\mathrm{RG})$ for $l=0$ on HN3 [30]. In Fig. 5, we compare the results for the packing fraction $\rho(\mu)$ as a function of the chemical potential for Wang-Landau sampling on networks with $N=2^{k}$ sites, $k=8 \sim 10$, with those from the exact RG after 500 iterations, corresponding to a system of $N=2^{500}$ sites. Despite the much smaller sizes of the Wang-Landau 
simulation, its results are barely distinguishable from the exact result, affirming the Wang-Landau sampling results as good references for our dynamic simulations, with negligible finite-size effects.

\section{B. Dynamic Properties}

The dynamic simulations of the BM on our networks uses the grand canonical partition function controlled by a chemical potential $\mu$ that mimics the experimental situation in a complex fluid or colloid, where particles are pumped into the larger system (the reservoir) and can enter the field-of-view through open boundaries inside a smaller window. For example, this could correspond to a $2 d$ slice of a $3 d$ colloidal bath used in colloidal tracking experiments [41]. Since our particles are not energetically coupled and merely obey hard excluded volume constraints, temperature is irrelevant and we can set $\beta=1$, making the chemical potential dimensionless, $\beta \mu \rightarrow \mu$. As we increase $\mu$, the system is more likely to accept more particles and increase the packing fraction $\rho(\mu)$. When $\mu$ is small (or negative), the reservoir and the network readily reach an equilibrium state with a certain packing fraction. However, when $\mu$ is large, the equilibrium state defined by the partition function has a packing fraction close to the close packing $\rho_{C P}$. Because of the density constraint and the disorder imposed by the hierarchical network geometry, the system enters into a jam at a density far from equilibrium packing. As in experiments, this jammed state remains for an extremely long time, even when $\mu$ is further increased. The ultimate packing fraction $\rho^{*}$ that the systems gets stuck at, in fact, is ever further from random close packing, the faster the quench in $\mu$ is executed, where $\frac{d \mu}{d t}$ is the quench rate. In this, our results closely resemble those reported in Ref. [23].

\section{Results for HN3}

The equilibrium packing fraction and entropy from Wang-Landau sampling as well as the dynamic results from simulated annealing for HN3 are shown in Fig. 6. Based on the analytical results by Boettcher et al. [30], we can confidently conclude that there is no phase transition in HN3 with $l=0$. Yet, the dynamic simulations indicate that the system jams nonetheless. The system jams even further from equilibrium for the case of $l=1$. Here, RG results have not been obtained so far and it is not clear whether there is a thermodynamic phase transition. The equilibrium results from Wang-Landau sampling (at $N=2^{10}$ ) seem to suggest a singularity near $1 / \mu \approx 0.06$ where the entropy density jumps noticeably and $\rho(\mu) \equiv \rho_{C P}$ for all larger $\mu$. Either RG or results for bigger systems may be needed to confirm whether there is phase transition or not.

The possible jamming transitions for both $l=0$ and 1 , revealed by the dynamic annealing simulations in Fig. 6
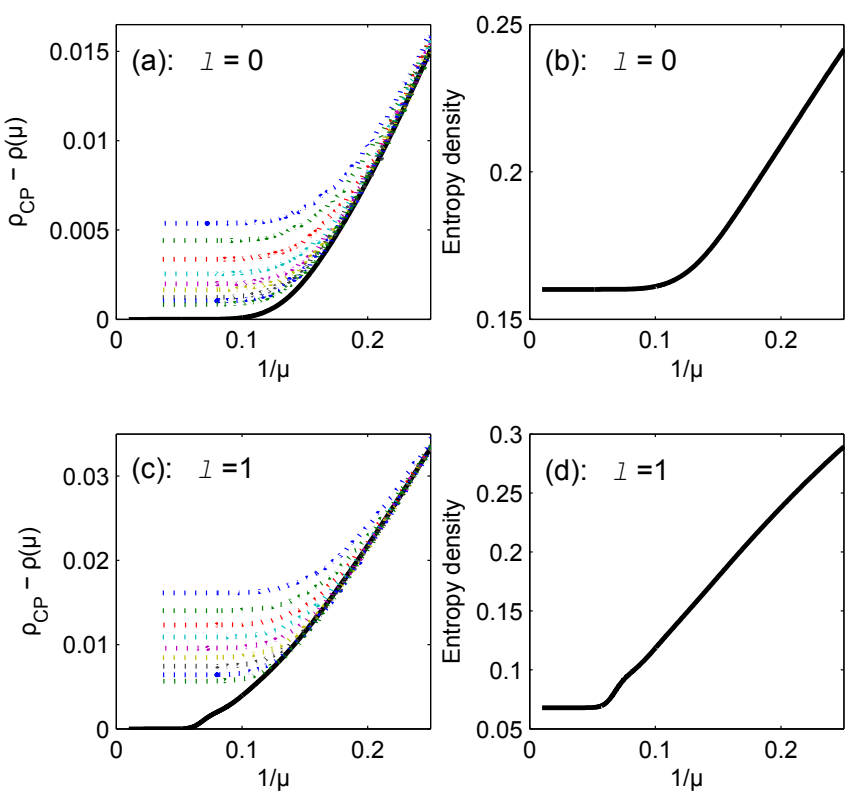

FIG. 6. Reduced packing fraction and entropy density for HN3 from Wang-Landau sampling and Simulated Annealing. (a) \&(b) are for $l=0$, and (c) \&(d) are for $l=1$. The black solid lines represent the equilibrium properties from WangLandau sampling with $N=1024$. The dotted lines are from simulated annealing with $N=32,768$, run at different annealing schedules with $d \mu=0.001 / 2^{j}$ for $j=0, \ldots, 8$, from top to bottom. Wang-Landau sampling provides the entropy density via Eq. (2), as shown in (b) and (d), which is difficult to obtain from other Monte Carlo methods. For both, $l=0$ and 1 , we find a non-zero entropy density for random close packing at $\mu \rightarrow \infty$.
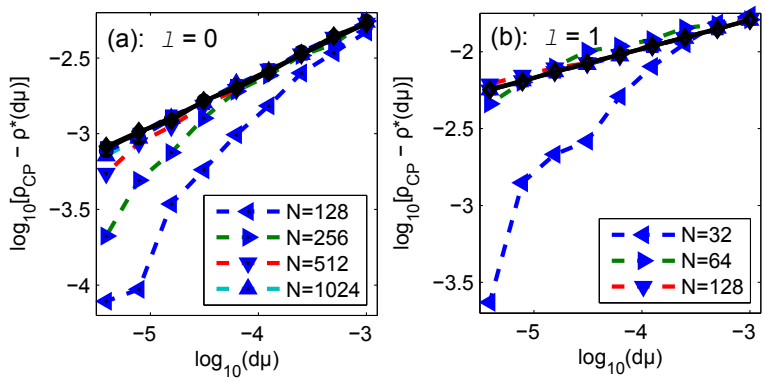

FIG. 7. Scaling of the dynamically reached packing fraction $\rho^{*}(d \mu)$ as a function of the annealing rate $d \mu$ for different system sizes $N$ of HN3. (a) For $l=0$, the dashed lines are for systems sizes $N=2^{k}$ with $k=7, \ldots, 10,12,14$ and 15 , from bottom to top. All data sets (except for the smallest sizes, $N=128, \ldots, 1024)$ collapse onto the top line with a slope of $0.34 \pm 0.01$, which is obtained from a fit using the data of the largest system size $N=32,768$. (b) For $l=1$, the data sets converge even faster towards power-law scaling. The dashed lines are for system sizes of $N=2^{k}$ with $k=5, \ldots, 8,10,12$, 14 and 15, from bottom to top. All but the first 3 sets collapse onto a line of slope $0.19 \pm 0.01$, which is obtained from a fit for $N=32,768$. Error bars are about of the size of each data point or smaller, indicating a relative error of less than $3 \%$. 


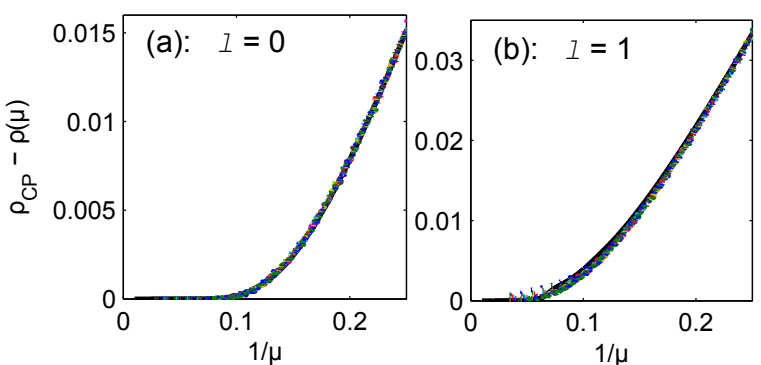

FIG. 8. Results of simulated annealing with hopping for HN3. For both (a) and (b), the figure consists of one solid line for the equilibrium result obtained with Wang-Landau sampling and 9 dotted lines obtained with simulated annealing at rates $d \mu=0.001 / 2^{j}$ for $j=0, \ldots, 8$. For HN3 with $l=0$ and $l=$ 1 , the system equilibrates for nearly all annealing schedules, collapsing the data onto the equilibrium line. Only for HN3 with $l=1$, small deviations from equilibrium are observed for annealing schedules $d \mu \gtrsim 10^{-5}$.

(a) and (c), are further supported by a power law decay of the residual packing fractions, $\rho_{C P}-\rho^{*}(d \mu)$, as a function of the annealing rate, $d \mu$. Here, we set the jammed packing fraction, obtained at $\mu \rightarrow \infty$ after annealing at rate $d \mu$, as $\rho^{*}(d \mu)=\rho(\mu \rightarrow \infty ; d \mu)$, where $d \mu / d t \rightarrow d \mu$ when measured in units of $d t \hat{=} 1$ sweep. Note that at these system sizes $(N=32,768)$, even the weakest jam is of order $\rho_{C P}-\rho^{*}(d \mu) \approx 0.001$ and, thus, still consists of a sizable number $(\gtrsim 30)$ of frustrated particles.

As shown in Fig. 7, a linear fit of the data on a doublelogarithmic scale at the largest systems is nearly perfect, justifying the assumption that the time-scales $1 / d \mu$ for the existence of the jam diverge asymptotically with a power law for $\rho \rightarrow \rho_{C P}$. For HN3 at $l=0$, the slope is $0.34 \pm 0.01$ with coefficient of determination $R^{2}=0.9975$, while for $l=1$ the slope is $0.19 \pm 0.01$ with $R^{2}=0.9997$, in both cases indicating a dramatic increase of timescales.

We also test the effect of introducing local hopping, implemented as suggested in step 3 of the algorithm in Sec. II D, which has not been addressed in Refs. [23, 40]. The results shown in Fig. 8 indicate a substantial difference from the simulation without hopping. For HN3 with $l=0$, the jamming transition disappears even for the fastest annealing schedule, $d \mu=10^{-3}$. For HN3 with $l=1$, the jamming transition can be eliminated at least for an annealing schedule of $d \mu \approx 10^{-5}$ or slower.

Besides the Hanoi networks, we have repeated the annealing simulations on random regular graphs, following Krzakala et al. [23]. On those graphs, BM with a hopping dynamics can reach a much denser state than with a varying chemical potential alone, which is similar to what Rivoire et al. [22] argue. But because of the enormous computational cost, we can only test $d \mu$ to as small as $\sim 10^{-6}$ for system sizes at most as large as $\sim 10^{5}$. No results are obtained to conclude that the jamming transition disappears entirely for some smaller $d \mu$, and we suspect that the behavior instead may resemble the
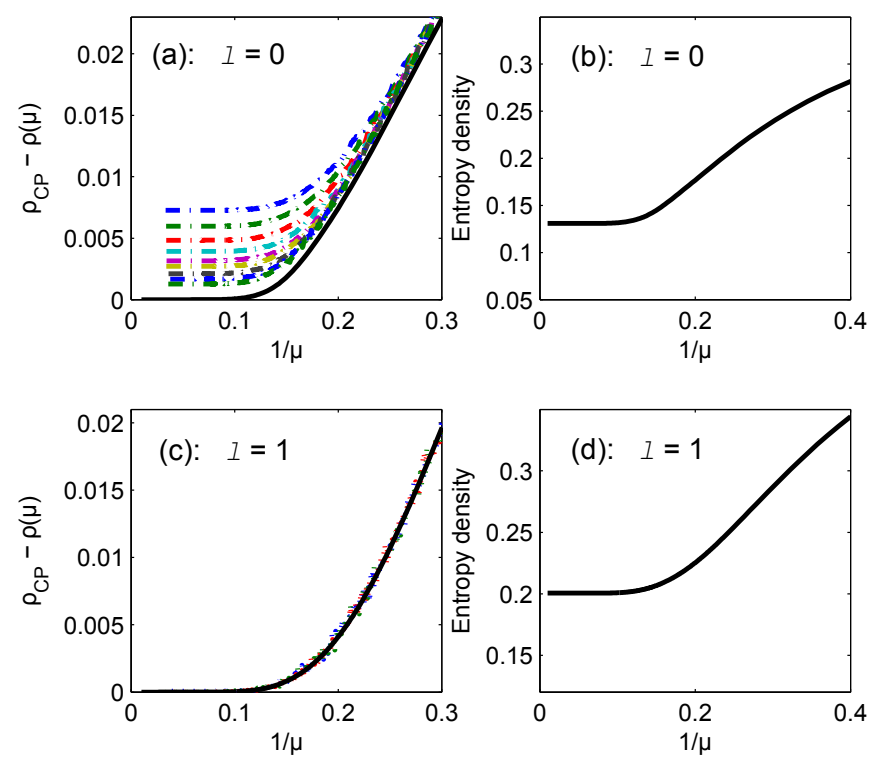

FIG. 9. Reduced packing fraction and entropy density for HN5 from Wang-Landau sampling and Simulated Annealing. (a) \&(b) are for $l=0$, and (c) \&(d) are for $l=1$. The black solid lines represent the equilibrium properties from WangLandau sampling with $N=1024$. The dotted lines are from simulated annealing with $N=32,768$, run at different annealing schedules with $d \mu=0.001 / 2^{j}$ for $j=0, \ldots, 8$, from top to bottom. As in Fig. 6, Wang-Landau sampling provides the entropy density via Eq. (2), as shown in (b) and (d).

mean-field predictions of Rivoire et al. [22].

\section{Results for HN5}

The case in HN5 is different from that in HN3. Note that HN5, unlike HN3 and most finite-dimensional lattices or the random graphs studied in Ref. [23], is not a regular network but has an exponential degree distribution. In HN5 for both, $l=0$ and $l=1$, as shown in Fig. 9, the equilibrium behavior obtained from WangLandau sampling is smooth and there is no indication of a phase transition. Annealing reveals a jamming transition and a power law decay similar to that in HN3 in the dynamic simulations only for $l=0$. For $l=1$, surprisingly, there is no jamming transition. The simulations with different annealing schedules equilibrate easily and collapse with the curves from Wang-Landau sampling. This suggests that the combination of heterogeneity in neighborhood sizes together with the possibility to have one occupied neighbor "lubricates" the system sufficiently to avert jams. Correspondingly, the results from WangLandau converge rapidly even for larger system sizes. As for HN3, permitting a local hopping dynamics unjams the system also for HN5 with $l=0$. 
TABLE II. Summary of the results. For each network, and the allowed neighborhood occupations of $l=0$ and $l=1$, we list to potential for a jam in dynamic simulations and the likely existence of an equilibrium glass transition.

\begin{tabular}{|c|c||c|}
\hline & $l=0$ & $l=1$ \\
\hline \hline HN3 & Jamming transition \& no phase transition & Jamming transition \& uncertain \\
\hline HN5 & Jamming transition \& uncertain & No jamming transition \& no phase transition \\
\hline HNNP & Jamming transition \& uncertain & No jamming transition\& no phase transition \\
\hline
\end{tabular}

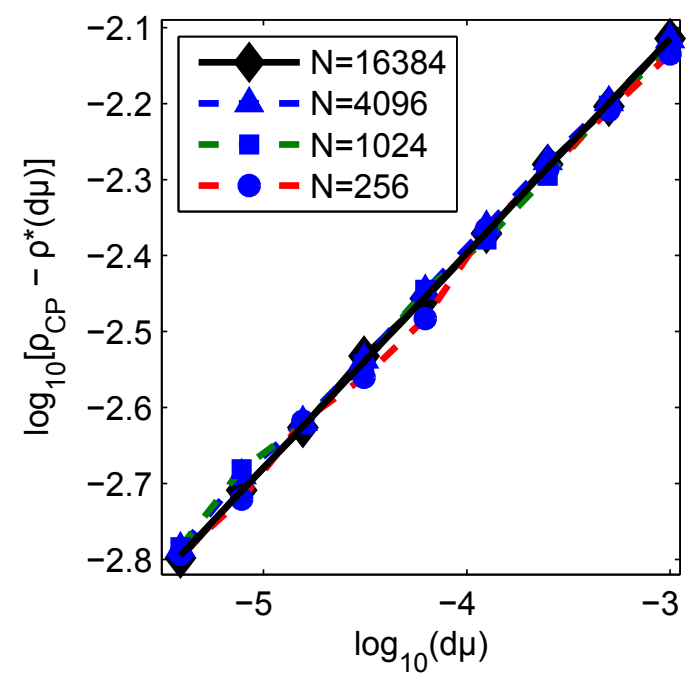

FIG. 10. Scaling of the dynamically reached packing fraction $\rho^{*}(d \mu)$ as a function of the annealing rate $d \mu$ for different system sizes $N$ of HN5 for $l=0$, the dashed lines are for systems sizes $N=2^{k}$ with $k=8,10,12$, and 14 . All data sets collapse onto the black solid line with a slope of $0.31 \pm 0.01$ with $R^{2}=0.9989$, which is obtained from a fit using the data of the largest system size $N=16,384$. Error bars are about of the size of each data point or smaller, indicating a relative error of less than $3 \%$.

\section{Results for HNNP}

HNNP provides an interesting alternative among the networks we are considering here. Unlike HN3 and HN5, HNNP is a nonplanar network, but like HN5 it has an exponential distribution of degrees with an average degree of 4. Most importantly, HNNP at $l=0$ possesses a "crystalline" optimal packing that is unique, see Fig. 11(b), and consists of every second site along the line being occupied, i.e., those sites that uniformly have the lowest degree of 3 . Therefore, it provides the opportunity to explore the potential for a first-order transition from a jammed state into the ground state, as was observed for lattice glasses in Ref. [25]. In this case, RG can be applied to obtain $\rho(\mu)$ in equilibrium exactly.

Indeed, we find a weakly jammed state in HNNP with $l=0$, with only a small number of frustrated particles, as shown in Fig. 11. The results of annealing simulations also show a power-law decay (Fig 12), consistent with the approach to a jamming transition. As RG suggest, and the smooth equilibrium curve for $N=1024$ and the
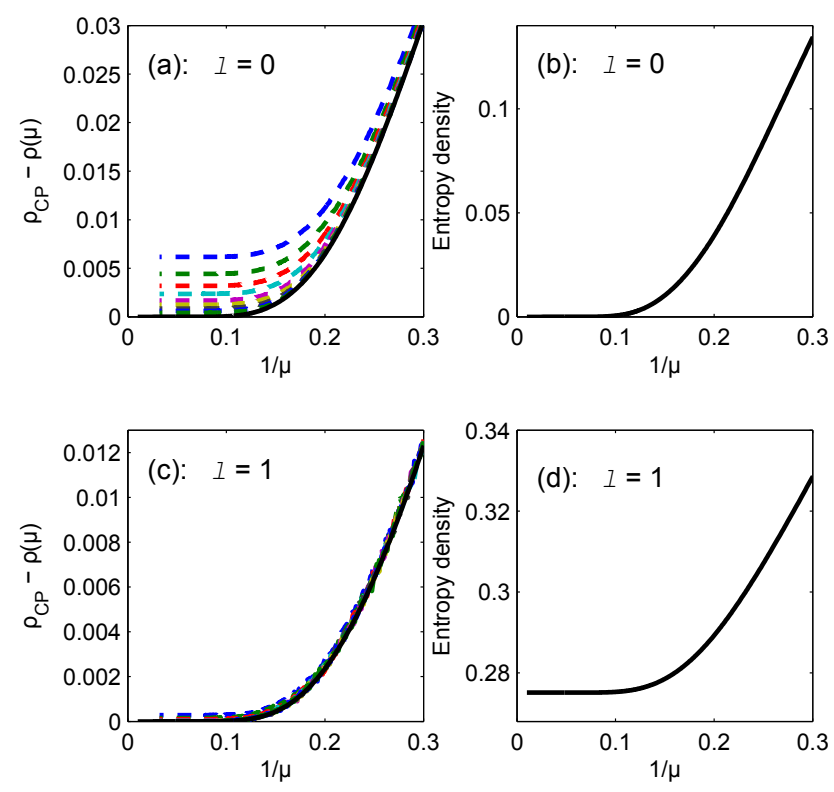

FIG. 11. Reduced packing fraction and entropy density for HNNP from Wang-Landau sampling and Simulated Annealing. (a) \&(b) are for $l=0$, and (c) \&(d) are for $l=1$. The black solid lines represent the equilibrium properties from Wang-Landau sampling with $N=1024$. The dotted lines are from simulated annealing with $N=16,384$, run at different annealing schedules with $d \mu=0.001 / 2^{j}$ for $j=0, \ldots, 8$, from top to bottom. As in Figs. 6 and 9, Wang-Landau sampling provides the entropy density via Eq. (2), as shown in (b) and (d). Note that in the limit of $\mu \rightarrow \infty$, HNNP at $l=0$ has a zero entropy which corresponds to a unique ground state. At $l=1$, it attains the same close packing fraction, $\rho_{C P}=\frac{1}{2}$, see Table I, but now at a non-trivial entropy.

convergence with increasing system sizes affirm, there is no thermodynamic phase transition in HNNP with $l=0$. Despite the weakness of those jams, we can find no indication that the annealing simulations at any rate $d \mu$ can ever decay into the ordered state. Apparently, the structural disorder, enforced in HNNP through a heterogeneous neighborhood degree and the hierarchy of longrange links, prevents such an explosive transition. The dominance of such structural elements is further emphasized by the fact that HNNP for $l=1$ exhibits no jams, similar to HN5, with which HNNP shares that structure. 


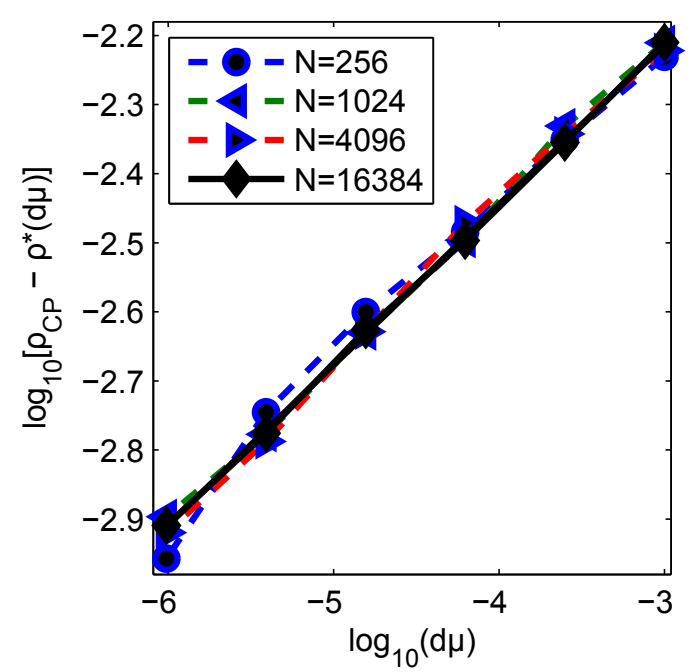

FIG. 12. Scaling of the dynamically reached packing fraction $\rho^{*}(d \mu)$ as a function of the annealing rate $d \mu$ for different system sizes $N$ of HNNP for $l=0$, the dashed lines are for systems sizes $N=2^{k}$ with $k=8,10,12$, and 14 . All data sets collapse onto the top line with a slope of $0.23 \pm 0.01$ with $R^{2}=0.9997$, which is obtained from a fit using the data of the largest system size $N=16,384$. Error bars are about of the size of each data point or smaller, indicating a relative error of less than $3 \%$.

\section{CONCLUSIONS}

We have examined the Biroli-Mezard lattice glass model on hierarchical networks, which provide intermediaries between solvable mean-field models and intractable finite-dimensional systems. These networks exhibit a lattice-like structure with small loops but also with a hierarchy of long-range links imposing geometric disorder and frustration while preserving a recursive structure that can be explored with exact methods, in principle. We observed a rich variety of dynamic behaviors in our simulations. For instance, we find jamming behavior on a regular network for which RG has shown that no equilibrium phase transition exists. However, whether the dynamic transition occurs at a packing fraction distinctly above random close packing remains unclear, and can only be resolved with more detailed RG studies that are beyond our discussion here.

We have simulated the model on our networks with a varying chemical potential $\mu$, with and without local hopping of particles. Hopping impacted those simulations in a significant manner, always eliminating any jams that have existed without hopping. Similar results has been found in mean-field systems in which the local dynamics can also affect the existence of jammed states [22]; however, the local dynamics may not eliminate the underlying phase transition in the mean-filed systems [22, 25]. Whether canonical simulations with hopping alone, or hopping at different rates, would change this scenario, we have to leave for future investigations, as well as the question on whether a combined method of updates would alter the behavior observed on lattices and mean-field networks.

\section{ACKNOWLEDGEMENTS}

We thank the U. S. National Science Foundation for its support through grant DMR-1207431.
[1] A. J. Liu and S. R. Nagel, Nature 396, 21 (1998).

[2] G. Biroli, Nature Physics 3, 222 (2007).

[3] A. J. Liu and S. R. Nagel, Annu. Rev. Condens. Matter Phys. 1, 347 (2010).

[4] L. Berthier and G. Biroli, Rev. Mod. Phys. 83, 587 (2011).

[5] C. S. O'Hern, S. A. Langer, A. J. Liu, and S. R. Nagel, Phys. Rev. Lett. 86, 111 (2001).

[6] L. Cipelletti and L. Ramos, Current Opinion in Colloid \& Interface Science 7, 228 (2002).

[7] L. Cipelletti and L. Ramos, Journal of Physics: Condensed Matter 17, R253 (2005).

[8] R. Hill and L. Dissado, Journal of Physics C: Solid State Physics 18, 3829 (1985).

[9] M. P. Ciamarra, M. Nicodemi, and A. Coniglio, Soft Matter 6, 2871 (2010).

[10] M. Van Hecke, Journal of Physics: Condensed Matter 22, 033101 (2010).

[11] A. Ikeda, L. Berthier, and G. Biroli, The Journal of Chemical Physics 138, 12A507 (2013).

[12] T. S. Majmudar, M. Sperl, S. Luding, and R. P. Behringer, Phys. Rev. Lett. 98, 058001 (2007).
[13] G. Parisi and F. Zamponi, Rev. Mod. Phys. 82, 789 (2010).

[14] L. Angelani and G. Foffi, Journal of Physics: Condensed Matter 19, 256207 (2007).

[15] V. Trappe, V. Prasad, L. Cipelletti, P. Segre, and D. Weitz, Nature 411, 772 (2001).

[16] H. Zhang and H. Makse, Physical Review E 72, 011301 (2005).

[17] L. Berthier, G. Biroli, J.-P. Bouchaud, L. Cipelletti, and W. van Saarloos, Dynamical heterogeneities in glasses, colloids, and granular media (Oxford University Press, 2011).

[18] F. Da Cruz, F. Chevoir, D. Bonn, and P. Coussot, Physical Review E 66, 051305 (2002).

[19] A. Ikeda, L. Berthier, and P. Sollich, Phys. Rev. Lett. 109, 018301 (2012).

[20] P. Charbonneau, J. Kurchan, G. Parisi, P. Urbani, and F. Zamponi, Nature Communications 5 (2014).

[21] P. Urbani and G. Biroli, arXiv preprint arXiv:1410.4523 (2014).

[22] O. Rivoire, G. Biroli, O. C. Martin, and M. Mézard, The European Physical Journal B-Condensed Matter and 
Complex Systems 37, 55 (2003).

[23] F. Krzakala, M. Tarzia, and L. Zdeborová, Phys. Rev. Lett. 101, 165702 (2008).

[24] H. Jacquin, L. Berthier, and F. Zamponi, Phys. Rev. Lett. 106, 135702 (2011).

[25] G. Biroli and M. Mezard, Phys. Rev. Lett. 88, 025501 (2002).

[26] W. Kob and H. C. Andersen, Phys. Rev. E 48, 4364 (1993).

[27] W. Kob and L. Berthier, Phys. Rev. Lett. 110, 245702 (2013).

[28] L. Berthier, Phys. Rev. E 88, 022313 (2013).

[29] S. Boettcher, B. Gonçalves, and H. Guclu, Journal of Physics A: Mathematical and Theoretical 41, 252001 (2008).

[30] S. Boettcher and A. K. Hartmann, Phys. Rev. E 84, 011108 (2011).

[31] S. Boettcher and C. T. Brunson, Phys. Rev. E 83, 021103 (2011).
[32] S. Boettcher, J. L. Cook, and R. M. Ziff, Phys. Rev. E 80, 041115 (2009).

[33] S. Boettcher, V. Singh, and R. M. Ziff, Nature Communications 3, 787 (2012).

[34] F. Wang and D. P. Landau, Phys. Rev. Lett. 86, 2050 (2001).

[35] F. Wang and D. P. Landau, Phys. Rev. E 64, 056101 (2001).

[36] H. K. Lee, Y. Okabe, and D. Landau, Computer physics communications 175, 36 (2006).

[37] A. Cunha-Netto and R. Dickman, Computer Physics Communications 182, 719 (2011).

[38] S. Kirkpatrick, C. D. Gelatt, and M. P. Vecchi, Science 220, 671 (1983).

[39] V. Černỳ, Journal of optimization theory and applications 45, 41 (1985).

[40] G. Biroli and M. Mézard, Phys. Rev. Lett. 88, 025501 (2002).

[41] G. L. Hunter and E. R. Weeks, Rep. Prog. Phys. 75, 066501 (2012). 